\title{
Identitätsmissbrauch im Internet
}

\section{Reinhold Sojer}

Dr., Wissenschaftlicher Mitarbeiter FMH eHealth

Beim Identitätsdiebstahl werden personenbezogene Daten missbräuchlich durch kriminelle Dritte entwendet und genutzt, um sich entweder finanziell zu bereichern oder um das Opfer gezielt zu schädigen. Da die Identität etwas ist, was im eigentlichen Sinne nicht gestohlen werden kann, wird richtigerweise der Begriff Identitätsmissbrauch verwendet. Dieser Begriff soll erstmals mit der Totalrevision des Bundesgesetzes über den Datenschutz und die Änderung weiterer Erlasse zum Datenschutz im Kontext des Ausbaus der strafrechtlichen Sanktionen im Schweizer Strafrecht aufgenommen werden.

Der Identitätsmissbrauch wird immer in zwei Schritten durchgeführt:

1. Zuerst erfolgt der Diebstahl von persönlichen Informationen,

2. danach werden die gestohlenen Informationen benutzt, um einen Missbrauch oder Betrug zu begehen.

Die persönlichen Informationen, die eine Person in der digitalen Welt identifizieren, werden durch Angreifer entweder ausgespäht oder durch Angriffe auf Kontendaten von Anbietern der Onlinedienste erbeutet. Für den Identitätsmissbrauch genügen bereits wenige Informationen wie Name, Adresse oder Geburtsdatum. Solche persönlichen Daten werden häufig bei der Registrierung von Onlinediensten, insbesondere Social Media, verlangt oder als Sicherheitsmerkmal bei telefonischen Verträgen zur Verifikation der Identität des Anrufers erfragt.

Für den Identitätsmissbrauch genügen bereits wenige Informationen wie Name, Adresse oder Geburtsdatum.

In welchem Ausmass Angreifer Daten erbeuten können, zeigt der Fall des Internetkonzerns Yahoo: Im August 2013 meldete Yahoo den Vorfall eines CyberAngriffs. 2017 wurde bekannt, dass bei diesem Angriff persönliche Informationen von mehr als 3 Milliarden Konten gestohlen wurden. Die Angreifer erhielten Zu-

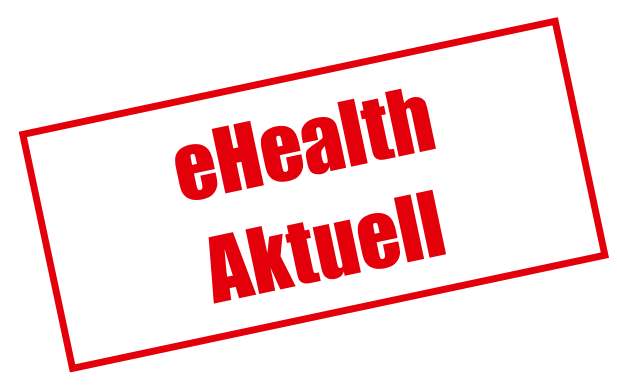

gang zu Daten wie Namen, E-Mail-Adressen oder Telefonnummern, die für weitere Angriffe von Interesse sind und die gezielt für einen Identitätsmissbrauch verwendet werden können.

Mit solchen gestohlenen Informationen werden im Namen des Opfers Waren bestellt oder falsche Informationen verbreitet, mit dem Ziel der Rufschädigung oder des Mobbings. Besonders beachtenswert ist der Anstieg der Entwendung von Gesundheitsdaten gemäss dem Breach Level Index (breachlevelindex.com), einer weltweiten Datenbank zur Registrierung von Datensicherheits- und Datenschutzverletzungen. Im Jahr 2015 wurde der zweitgrösste Krankenversicherer in den USA Anthem Opfer eines Cyber-Angriffes, bei dem Daten von 78 Millionen Versicherten gestohlen wurden. Auch wenn keine medizinischen Daten direkt betroffen waren, so liegt das Schadenspotential im Versicherungsbetrug oder in der Erpressung anhand persönlicher Informationen. Das höchste Schadenspotential entsteht jedoch dann, wenn Angreifer zusätzlich in Kenntnis von Passwörtern gelangen.

\section{Phishing $=$ Password + Harvesting + Fishing}

Um an geheime Informationen zu gelangen, werden Methoden des Social Engineering eingesetzt, welche darauf abzielen, die Hilfsbereitschaft, Gutgläubigkeit oder die Unsicherheit von Personen auszunutzen. Eine häufige Methode zum Erspähen von Passwörtern ist Phishing. Phishing ist ein Kunstwort aus Password, Harvesting und Fishing und täuscht eine Person mit gefälschten E-Mails, Websites oder Twitternachrichten, um in den Besitz von persönlichen Login-Daten zu gelangen. Als Absender wird eine namhafte Organisation oder im Fall eines finanziellen Betruges eine Bank angegeben. Aber auch Schadprogramme können dazu verwendet werden, um eine Person auf eine böswillige Website zu führen (z.B. durch Browser-Hijacker). Hat es der Phisher auf Zugangsdaten für Onlinedienste abgesehen, sendet er ein Spam-E-Mail ungezielt an möglichst viele Empfänger und spekuliert darauf, dass unter den vielen Empfängern auch Kunden des ent- 
sprechenden Onlinedienstes, auf die er es abgesehen hat, reagieren. Effizienter ist das Versenden von betrügerischen E-Mails unter Verwendung gekaufter oder gehackter Adressen wie im Fall des Internetkonzerns Yahoo. Der Empfänger wird nun im nächsten Schritt innerhalb der gefälschten Website aufgefordert, sich mit seinem Benutzernamen und seinem Passwort auszuweisen, und somit gelangen diese Daten in die

\section{Anwendungen wie Twitter, WhatsApp, Face- book, Instagram oder Google Translate sam- meln unentwegt persönliche Informationen.}

Hände der Cyber-Kriminellen. Seit einigen Jahren werden Phishing-Angriffe auch mit Ransomware-Schadsoftware kombiniert (siehe SÄZ 2016;97(49-50):1708-9). Eine Sonderform des Phishings ist das sogenannte Spear-Phishing, bei dem der Angreifer nicht nur die EMail-Adresse des Opfers kennt, sondern auch Details aus dem privaten und beruflichen Umfeld. So kann der Angreifer im Namen einer vertrauenswürdigen Person oder Organisation mit einer plausiblen Aufforderung etwa per E-Mail oder über Facebook dazu bewegen, eine bestimmte Aktion auszuführen. Mit diesem Vorgehen wurden im Sommer 2016 in Deutschland gezielt Nachrichten mit dem gefälschten Absender hq.nato.int an Politiker versendet, welche Informationen zum Militärputsch in der Türkei enthielten und unter anderem auch einen Link zu einer mit Schadcode infizierten Website.

Je mehr persönliche Daten über das Opfer ausgespäht werden können, desto authentischer kann die betrügerische Nachricht gestaltet werden. Anwendungen wie Twitter, WhatsApp, Facebook, Instagram oder Google Translate sammeln unentwegt persönliche Informationen, haben Zugriff auf Kontaktdaten, Kalender oder den Standort und: Sie wissen am besten, wer Sie sind.

\section{Wie kann man sich schützen?}

Korrespondenz: Dr. rer. biol. hum Reinhold Sojer Wissenschaftlicher Mitarbeiter / Stv. Leiter Abteilun Digitalisierung / eHealth FMH Verbindung der Schweizer Ärztinnen und Ärzte

Elfenstrasse 18

Postfach 300

3000 Bern 15

Telefon: +413135912 04

reinhold.sojer[at]fmh.ch

Gegen einen Identitätsmissbrauch gibt es keinen hundertprozentigen Schutz. Dies liegt einerseits daran, dass persönliche Daten in diversen Institutionen (Banken, Versicherungen, Spitäler usw.) gespeichert werden und auf diese nur noch wenig Einfluss genommen werden kann. Andererseits gehen Cyber-Kriminelle bei vor, und die in diesem Artikel benannten Methoden stellen nur ein kleines Spektrum derer dar, die heute genutzt werden. Auch werden sie kombiniert angewen- det mit Methoden jenseits der digitalen Welt, wie zum Beispiel dem Dumpster Diving (Durchsuchen von Abfällen nach persönlichen Informationen).

- Machen Sie sich mit der Thematik Identitätsmissbrauch vertraut und setzen Sie sich damit auseinander. Die richtige Awareness schützt bereits nachhaltig.

- Schützen Sie sich vor Schadsoftware (Malware), indem Sie Ihre Systeme stets auf den neusten Stand halten und Sicherheitsupdates umgehend installieren (siehe Empfehlungen aus SÄZ 2016;97(49-50): 1708-9).

- Geben Sie personenbezogene Daten äusserst «sparsam» und nur auf vertrauenswürdigen Websites und Portalen ein. Verwenden Sie nie Ihr reales Geburtsdatum in sozialen Netzwerken

- Misstrauen Sie E-Mails, die Sie unaufgefordert bekommen, und seien Sie vorsichtig, wenn Sie E-Mails erhalten, die eine Aktion von Ihnen verlangen und andernfalls mit Konsequenzen drohen.

- Öffentliche WLANs bergen die Gefahr des Abhörens durch Dritte. Wenn Sie sensitive Informationen austauschen, sollten Sie diese durch Nutzung einer zusätzlichen Verschlüsselungsebene (HTTPS, VPN oder spezielle Browser-PlugIns) schützen.

- Verwenden Sie keinesfalls stets die gleichen Passwörter für verschiedene Internetkonten und ändern Sie diese von Zeit zu Zeit. Die Verwendung verschiedener Benutzernamen für Onlinedienste erschwert Betrügern, ein Gesamtprofil Ihrer Identität zu erstellen.

\section{Gegen einen Identitätsmissbrauch gibt es keinen hundertprozentigen Schutz.}

- Nutzen Sie wo immer möglich die Zwei-FaktorAuthentifizierung (2FA). Bei der 2FA wird unter anderem neben Benutzername und Passwort ein Nachweis in Form eines Besitzes verlangt. Dies kann eine TAN-Liste oder ein Security Token wie eine Smartcard sein, welche ausschliesslich im Besitz des Kontoinhabers ist. der Beschaffung von Identitäten meist professionell
- Verwenden Sie eine adäquate Endpoint-SecuritySoftware (Virenscanner, Firewall usw.) und halten Sie diese aktuell.

Recherchieren Sie proaktiv im Internet, welche Daten von Ihnen gespeichert sind, und überprüfen Sie, ob diese korrekt sind. Unter der Adresse https:// haveibeenpwned.com können Sie überprüfen, ob Ihre E-Mail-Adresse oder Benutzerkonto kompromittiert wurde. 